\title{
Neurofibromas of the bladder in a child with neurofibromatosis type 1
}

\author{
Gulec Mert Dogan ${ }^{1}$, Ahmet Siğirci ${ }^{1}$, Leyla Karaca ${ }^{1}$ \\ ${ }^{1}$ Department of Radiology Pediatric, Inonu University Malatya, Turkey
}

\section{CASE DESCRIPTION}

A 17-year-old boy diagnosed with neurofibromatosis type 1 (NF1) presented with a six-month history of hematuria, dysuria, and urinary frequency. Ultrasonography (USG) revealed diffuse thickening of the anterosuperior and posterior walls of the bladder with round, $<5 \mathrm{~mm}$ nodular echogenities in the thickened walls (Figure-1). Magnetic resonance imaging (MRI) of the pelvis revealed a nodular lesion with low signal intensity on T1 and fat suppressed T1 weighted (T1-W) images; and nodular lesions with a 'target sign' on T2 weighted (T2-W) images. This consisted of low signal intensity fibrosis surrounded by high signal intensity stroma at the posterior of the bladder wall (Figures 2 and 3). The patient's symp-

Figure 1 - Ultrasound imaging; Diffuse thickening of the anterosuperior and posterior walls of the bladder and multiple round, $<5 \mathrm{~mm}$ nodular echogenities in the thickened walls marked with arrows.

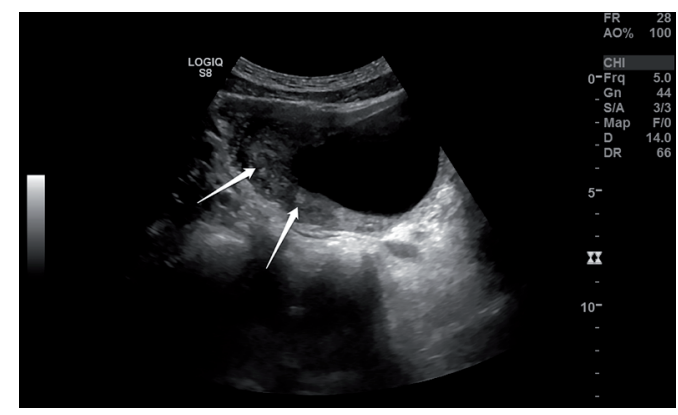

Figure 2 - MRI axial images; nodular lesions marked with arrows. A. T1 weighted B. post contrast T1 weighted fat suppressed C. T2 weighted fat suppressed image.

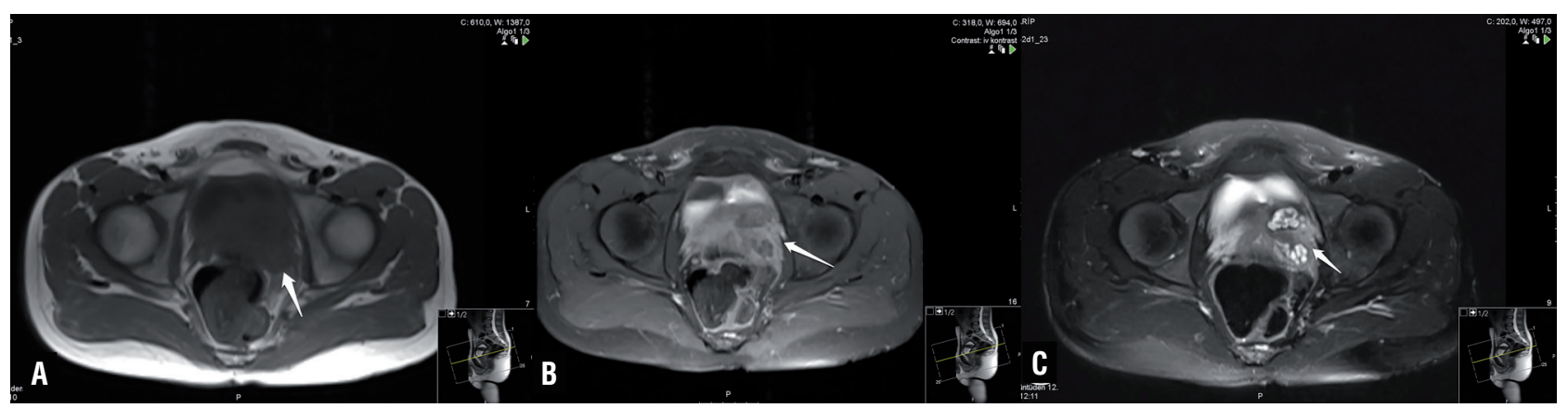


Figure 3 - MRI sagittal images; nodular lesions marked with arrows A. T1 weighted fat suppressed B. Post contrast T1 weighted fat suppressed C. T2 weighted image.

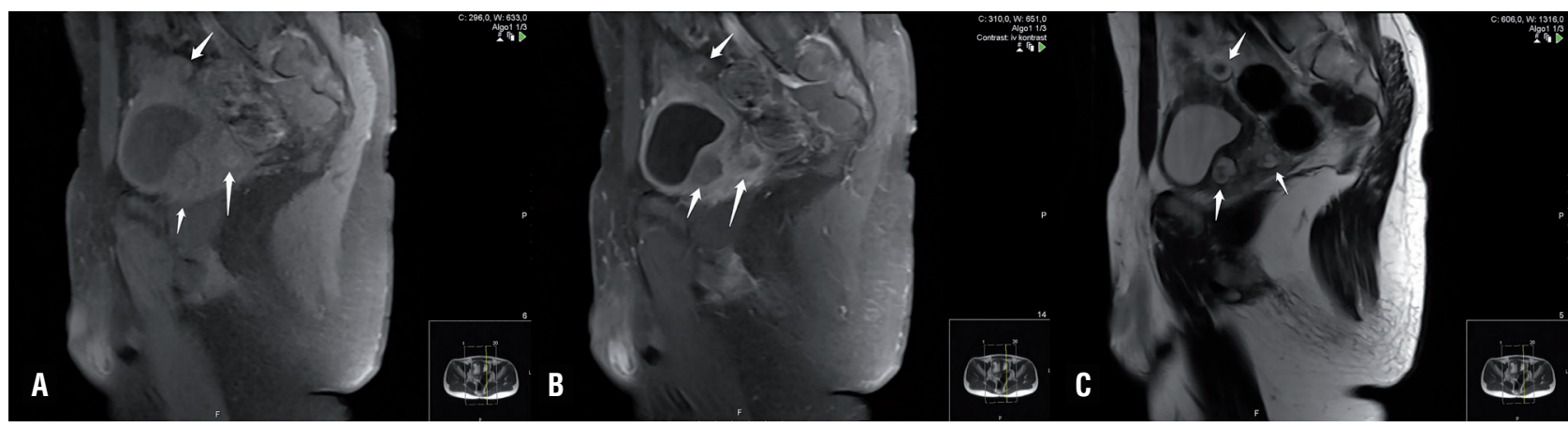

toms were relieved after antibiotic treatment and he has had no serious complaints since then. He is now monitored by the urology outpatient clinic.

Children with NF1 should always be evaluated for neurofibromatosis of the genitourinary system (1). Bladder involvement of neurofibromatosis is rare and presenting features include irritative voiding symptoms and hematuria due to recurrent urinary tract infections (2). On USG, bladder involvement of neurofibromas can manifest as a focal mass or as diffuse bladder wall thickening. On MRI, neurofibromas display low-signal intensity on T1-W images and non-homogeneous high-signal intensity with a 'target sign' on T2-W images (3). Differential diagnosis includes rhabdomyosarcoma, ganglioneu-

\section{REFERENCES}

1. Yılmaz K, Dusunsel R, Dursun I, Coskun A, Erten S, Kucukaydın M, et al. Neurofibromas of the bladder in a child with neurofibromatosis type 1 causing chronic renal disease. Ren Fail. 2013;35:1005-7.

2. Meesa IR, Junewick JJ. Pelvic plexiform neurofibroma involving the urinary bladder. Pediatr Radiol. 2008;38:916.

3. Wilkinson LM, Manson D, Smith CR. Best cases from the AFIP: plexiform neurofibroma of the bladder. Radiographics. 2004;24 Suppl 1:S237-42.

4. Smirniotopoulos JG, Lonergan GJ, Abbott RM, Choyke PL, Czum JM, Dalrymple NC, et al. Image interpretation session: 1998. The Radiological Society of North America 84th scientific assembly and annual meeting. Radiographics. 1999;19:205-33. roma, and retroperitoneal fibrosis (4). In a patient with NF1, the primary consideration should be neurofibroma. Generally, management of patients with NF1 and bladder involvement is conservative. If there are intractable symptoms such as hydronephrosis, bladder volume loss and suspicion for malignant degeneration, surgical treatment may be needed (3).

In conclusion, conventional MRI and ultrasound are important imaging modalities for the evaluation of genitourinary involvement of neurofibromatosis disease type 1 .

\section{CONFLICT OF INTEREST}

None declared.

\author{
Correspondence address: \\ Gulec Mert Dogan, MD \\ Department of Radiology Pediatric \\ Inonu University Malatya \\ Malatya 44280, Turkey \\ E-mail: dr_gulecmert@hotmail.com
}

\section{ARTICLE INFO}

Int Braz J Urol. 2018; 44: 1256-7

Submitted for publication: April 05, 2017

Accepted after revision: December 27, 2017

Published as Ahead of Print: February 22, 2018 